Pacific Journal of Mathematic 


\section{PROPERTIES PRESERVED UNDER HOMOMORPHISM}

\section{ROGER C. LYNDON}

1. Introduction. The main result of this paper is a characterization of those sentences of the predicate calculus whose validity is preserved under passage from an abstract algebraic system to any homomorphic image of the system. An algebraic system is here construed to be a set together with certain operations and relations, including identity, defined for elements of the set. The sentences under consideration will contain symbols for these operations and relations, and variables whose range is the set of elements of the system, together with the usual logical symbols, but will contain no variables whose range consists of sets, relations, or functions. Such a sentence will be called positive if it contains the logical symbols for conjunction, disjunction and quantification only, but not the symbol for negation. It will be shown that:

(*) A sentence of the predicate calculus is preserved under homomorphism if and only if it is equivalent to a positive sentence.

An example is provided by the usual statement of the commutative law for multiplicative systems:

$$
\forall x y \cdot x y=y x .
$$

This is a positive sentence, and indeed every homomorphic image of a commutative system in commutative. As a second example, upon eliminating the symbol for "if ... then", the left cancellation law takes the form

$$
\forall x y z \cdot \sim(x y=x z) \vee y=z .
$$

This sentence is not positive, and, indeed, from the fact that the left cancellation property is not preserved under homomorphism we conclude that it is not expressible by any positive sentence.

It is not difficult to show that every sentence equivalent to a positive sentence is preserved under homomorphism; although the converse seems nearly as obvious intuitively, to prove the converse appears to be a matter of considerable difficulty. That positive sentences are preserved was noted by the author [6], and also by E. Marczewski [9], who raised the question of the converse. A proof, by methods quite different from those used here, was announced by J. Eoś [5], but such a proof has not been published. The result has also been stated by A. I. Malcev [8], who appears to indicate a method of proof.

Received October 22, 1958. Work supported in part under grants from the National Science Foundation. 
The central result of this paper, Theorem 5, is in fact a stronger form of the assertion $(*)$ above. Some consequences and variants of this theorem are given, and examples to show that it can not be strengthened further in certain obvious ways.

The content of this paper lies within the theory of elementary classes as formulated by A. Tarski $[12,13]^{1}$. We define and use here numerous concepts due to him, and, in particular, that of elementary extension, due to Tarski and R. Vaught [15]. We have tried to make this paper self contained to the extent that the main line of reasoning should be intelligible and convincing under any reasonable interpretation of the concepts employed; for the technical definitions necessary for rigor in the details of the proofs, we refer to an earlier paper [7]. Further, we borrow from that paper the relevant definitions and a proof for the following theorem, which is the cornerstone of the present paper:

INTERPOLATION THEOREM. If $S$ and $T$ are sentences of the predicate calculus, and $S$ implies $T$, then there exists a sentence $M$ such that $S$ implies $M$ and $M$ implies $T$, and that a relation symbol occurs positively (negatively) in $M$ only if it occurs positively (negatively) in both $S$ and $T$.

The author has profited from many discussions with L. Henkin and A. Tarski. ${ }^{2}$ The relativization embodied in Theorem $5^{\prime}$ was suggested by A. Robinson. ${ }^{3}$

2. Sentences increasing in a relation symbol. Roughly, a property of a relation may be called increasing if, whenever it holds for a given relation it holds for any larger relation. Passing from properties to the sentences that express them, we make a precise definition. Let $Q$ be a subset of the set $R$ of all relation symbols in a language $L$, and let $Q^{\prime}$ be a set of new and distinct relations symbols $q^{\prime}$ in one-to-one correspondence with the symbols $q$ of $Q$ in such a way that $q^{\prime}$ has the same rank as $q$. Let $I$ be the set of all sentences

$$
I\left(q, q^{\prime}\right)=\forall x_{1} \cdots x_{\rho(q)} \cdot q\left(x_{1}, \cdots, x_{\rho(q)}\right) \supset q^{\prime}\left(x_{1}, \cdots, x_{\rho(q)}\right),
$$

for all $q$ in $Q$. Let $I^{\prime}$ be a set of formulas of $L$, and $I^{\prime}$ the result of replacing the symbols $q$ in $I^{\prime}$ by the corresponding $q^{\prime}$. We call $\Gamma$ increasing in $Q$ if $\Gamma^{\prime}, I \Rightarrow \Gamma^{\prime}$.

1 We use the word 'elementary' in preference to 'arithmetical', and, by an 'elementary class', mean always what is commonly called an 'arithmetical class in the wider sense $\left(A C_{\Delta}\right)^{\prime}$.

2 In particular, while the author was visiting at the University of California, Berkeley.

3 At the American Mathematical Society Summer Institute, Ithaca, 1957. 
Proposition 1. If a set $\Gamma$ of formulas is positive in all the relation symbols in a set $Q$, then $\Gamma$ is increasing in $Q$.

Proof. It suffices to treat the case that $\Gamma$ consists of a single formula $F$. If $F$ is an atomic formula or, vacuously, the negation of an atomic formula, the conclusion is immediate. The general case follows by an obvious induction.

The converse is contained in the following.

Proposition 2. Let $L, Q, Q^{\prime}$ and $I$ be as before. Let $\Sigma, \Gamma, \Delta$ be sets of sentences $L$, and let $\Sigma^{\prime}$ result from $\Sigma$, and $\Delta^{\prime}$ from $\Delta$, by replacing each $q$ by the corresponding $q^{\prime}$. If $\Sigma, \Sigma^{\prime}, \Gamma, I \Rightarrow \Delta^{\prime}$, then there exists a set $I$ of sentences $P$, positive in all the symbols of $Q$ and not containing the symbols of $Q^{\prime}$, such that $\Sigma, \Gamma \Rightarrow \Pi$ and $\Sigma, \Pi \Rightarrow \Delta$.

Proof. It suffices to treat the case that $\Delta$ consists of a single sentence $D$. By the Compactness Theorem (Corollary 4.1 of [1]), the hypothesis will hold with $\Sigma, \Gamma, I$ replaced by finite subsets, and hence, taking conjunctions, by single sentences: $S, S^{\prime}, C, J \Rightarrow D^{\prime}$, where $C$ is positive in all the $q$ in $Q$, and $J$ is a conjunction of sentences $I\left(q, q^{\prime}\right)$. It follows directly that $S, C \Rightarrow J \wedge S^{\prime} \supset D^{\prime}$. The symbols $q^{\prime}$ do not occur at all in $S$ or $C$. The symbols $q$ occur only in the part $J$ of $J \wedge S^{\prime} \supset D^{\prime}$, and since each occurrence of a symbol $q$ is negative in $J$, it is positive in $J \wedge S^{\prime} \supset D^{\prime}$. By the Interpolation Theorem there exists a sentence $P$, not containing the $q^{\prime}$ and positive in the $q$, such that $S, C \Rightarrow P$ and $P \Rightarrow J \wedge S^{\prime} \supset D^{\prime}$. From $S, C \Rightarrow P$ we have $\Sigma, \Gamma \Rightarrow P$. From $P \Rightarrow J \wedge S^{\prime} \supset D^{\prime}$, replacing each $q^{\prime}$ by $q$, it follows that $P \Rightarrow J^{*} \wedge S \supset D$ where $J^{*}$ is the result of replacing each $q^{\prime}$ by $q$ in $J$. In fact, $J^{*}$ is a theorem, whence $P \Rightarrow S \supset D$, hence $P, S \Rightarrow D$, and $\Sigma, P \Rightarrow D$.

CoROllary 2.1. A set $I$ of sentences is increasing in the symbols of $Q$ if and only if it is equivalent to a set $I I$ of sentences positive in the symbols of $Q$.

3. Q-maps. If $\Gamma$ is a set of sentences of the language $L$, let $\Gamma^{*}$ be the set of all models of $L$ in which all sentences of $\Gamma$ hold. If $K$ is a set of models of $L$, let $K^{*}$ be the set of all sentences of $L$ that hold in all models in $K$. It follows that $\Gamma^{* *}$ is the 'logical closure' of $\Gamma$, the set of all sentences $S$ such that $\Gamma \Rightarrow S$. The elementary closure of $K$ is $K^{* *}$, and $K$ is an elementary class if $K=K^{* *}$, that is, if $K=\Gamma^{*}$ for any $\Gamma$. Two models $\mathfrak{A}$ and $\mathfrak{B}$ are elementarily equivalent if $\mathfrak{A}^{*}=\mathfrak{B}^{*}$, that is, if exactly the same sentences hold in $\mathfrak{A}$ as in $\mathfrak{B}$.

A model $\mathfrak{A}$ is submodel of a model $\mathfrak{B}$ if the domain $A$ of $\mathfrak{A}$ is a subset of the domain $B$ of $\mathfrak{B}$ and if each $\mathfrak{A} w, \mathfrak{A} r$ is the restriction of 
the corresponding $\mathfrak{B} w, \mathfrak{B} r$ to the subset $A$ of $B$. If $\mu$ is any interpretation in $\mathfrak{A}$, there is a unique interpretation $\lambda$ in $\mathfrak{B}$ such that $\mu$ and $\lambda$ agree on all variables of $L$. $\mathfrak{B}$ is an elementary extension of $\mathfrak{A}$ if for all $\mu, \lambda$ as above, and $F$ a formula of $L$, if $F$ holds in $\mu$ then $F$ holds in $\lambda$. In particular, $\mathfrak{A}$ and $\mathfrak{B}$ are elementarily equivalent.

If $\mathfrak{A}$ and $\mathfrak{B}$ are models with domains $A$ and $B$, a map $\theta$ of $A$ onto $B$ will be called a $Q-m a p$, for $Q \subseteq R$ a set of relation symbols, if, first of all, $\theta$ 'preserves' all operations and relations:

$$
\begin{aligned}
& \theta\left[(\mathfrak{A} w)\left(a_{1}, \cdots, a_{\rho(w)}\right)\right]=(\mathfrak{B} w)\left(\theta a_{1}, \cdots, \theta a_{\rho(w)}\right), \quad \text { all } w \text { in } W, \\
& (\mathfrak{A} r)\left(a_{1}, \cdots, a_{\rho(r)}\right) \Rightarrow(\mathfrak{B} r)\left(\theta a_{1}, \cdots, \theta a_{\rho(r)}\right), \quad \text { all } r \text { in } R \text {, }
\end{aligned}
$$

and, moreover, the implication in the last line is an equivalence for all $r$ not in $Q$. More concisely, $\theta(\mathfrak{A} w)=\mathfrak{B} w, \theta(\mathfrak{A} r) \subseteq \mathfrak{B} r$, with $\theta(\mathfrak{A} r)=\mathfrak{B} r$ for $r$ not in $Q$. If $\theta$ is one-to-one, we speak of a $Q$-isomorphism. An $O$-isomorphism, for $\mathrm{O}$ the empty set, is an isomorphism in the usual sense. ${ }^{4}$

If $\theta$ is any map of $\mathfrak{A}$ onto $\mathfrak{B}$, its kernel $k$, defined by $k\left(a, a^{\prime}\right)$ if and only if $\theta a=\theta a^{\prime}$, is an equivalence relation on $A$. If $\theta$ is a O-map of $\mathfrak{A}$ onto $\mathfrak{B}$, then $k$ is a congruence relation on $\mathfrak{A}$, that is, it is substitutive with respect to all the $\mathfrak{A} w$ and $\mathfrak{A} r$. For any congruence $k$ on a model $\mathfrak{A}$, the operations $\mathfrak{A} w$ and relations $\mathfrak{A} r$ of $\mathfrak{A}$ induce operations $\mathfrak{A} w / k$ and relations $\mathfrak{A} r / k$ on the set $A / k$ of equivalence classes in $A$ under $k$; the quotient model $\mathfrak{A} / k$ is defined to have domain $A / k$, operations $(\mathfrak{U} / k) w=\mathfrak{A} w / k$, and relations $(\mathfrak{A} / k) r=\mathfrak{A} r / k$. It is immediate that the natural projection of $A$ onto $A / k$ is a $O$-map, and that if $\theta$ is any $O$-map of $\mathfrak{A}$ onto $\mathfrak{B}$, with kernel $k$, then $\theta$ induces a naturally an isomorphism of $\mathfrak{A} / k$ onto $\mathfrak{B}$.

We proceed to the statement of a proposition that contains all that we require about elementary extensions. For $\mathfrak{A}$ a model of the language $L$, with domain $A$, define a language $L_{A}$ by adjoining to $L$ new and distinct constants (operations of rank 0 ) $w_{a}$ for all $a$ in $A$, and a new relation $e_{A}$ of rank two. Extend $\mathfrak{A}$ to a model $\mathfrak{A}_{A}$ of $L_{A}$ by defining $\mathfrak{A}_{A} w_{a}=a$, that is, $\mathfrak{A}_{A} w_{a}$ is the constant operation with value $a$, and $\mathfrak{A}_{A} e_{A}$ to be the identity relation on $A$. Then $\mathfrak{H}_{A}^{*}$ is the set of all sentences of $L_{A}$ that hold in $\mathfrak{A}_{A}$.

Proposition 3. If $\mathfrak{U}$ and $\mathfrak{B}$ are models of $L$, and $\mathfrak{B}$ has an extension $\mathfrak{B}^{\prime}$ to $L_{4}$ in which all sentences of $\mathfrak{U}_{A}^{*}$ hold, then $\mathfrak{B}$ has an O-image that is an elementary extension of $\mathfrak{A}$.

4 The concept of $Q$-map and that of elementary extension, as well as various results mentioned here, are special cases of more far-reaching ideas developed recently by $\mathrm{H}$. J. Keisler [4]. The use of constants $w_{a}$, in the paragraph after next, derives from the 'diagrams' of A. Robinson [11]. Proposition 3 in contained in Th. 1.11 of [15]. 
Proof. Let $k=\mathfrak{B}^{\prime} e_{A}$. Since the sentences expressing that $\mathfrak{A}_{A} e_{A}$, the identity on $A$, is a congruence on $\mathfrak{A}_{A}$ are in $\mathfrak{U}_{A}^{*}$, they hold in $\mathfrak{B}^{\prime}$, whence $k$ is a congruence on $\mathfrak{B}^{\prime}$. The quotient system $\mathbb{C}^{\prime}=\mathfrak{B}^{\prime} / k$ then also satisfies $\mathfrak{U}_{A}^{*}$, and $\mathbb{C}^{\prime} e_{A}$ is the identity on the domain $C$ of $\mathbb{C}^{\prime}$. The restriction $\sqrt{5}$ of $\mathfrak{S}^{\prime}$ to $L$ is $\mathfrak{B} / k$, an O-image of $B$.

Define a $\operatorname{map} \theta$ from $A$ into $C$ by setting $\theta a=\mathbb{C}^{\prime} w_{a}$. Now, $\mathfrak{U}_{A} w\left(a_{1}, \cdots, a_{\rho(w)}\right)=a^{\prime}$ if and only if $e_{A}\left(w\left(w_{a_{1}}, \cdots, w_{a_{\rho(w)}}\right), w_{a^{\prime}}\right)$ is in $\mathfrak{A}_{A^{*}}$, hence if and only if this sentence holds in $\mathbb{C}^{\prime}$, that is, if

$$
\mathfrak{S}^{\prime} w\left(\theta a_{1}, \cdots, \theta a_{\rho(w)}\right)=\theta a^{\prime} \text {. }
$$

The same reasoning shows that $\mathfrak{A}_{A} r\left(a_{1}, \cdots, a_{\rho(r)}\right)$ holds in $\mathfrak{A}$ if and only if $\mathbb{C}^{\prime} r\left(\theta a_{1}, \cdots, \theta a_{\rho(r)}\right)$ in $\mathfrak{C}^{\prime}$. This establishes that is an 0 -map of $\mathfrak{A}_{A}$ onto a subsystem $\theta \mathfrak{A}_{A}$ of $\mathcal{S}^{\prime}$, and, in fact, taking $r$ above to be $e_{A}, a=a^{\prime}$ if and only if $\theta a=\theta a^{\prime}$, whence $\theta$ is an isomorphism.

Since $\theta \mathfrak{A}_{A}$ is a submodel of $\mathfrak{C}^{\prime}$, taking restrictions to $L, \theta \mathfrak{A}$ is a submodel of $\sqrt{5}$. Let $\mu$ be an interpretation in $\theta \mathfrak{A}$, and $\lambda$ the interpretation in $\sqrt{5}$ that agrees with $\mu$ on all variables. Let $F$ be a formula of $L$ with free variables $x_{1}, \cdots, x_{n}$, and $D$ the sentence that results from $F$ by replacing each $x_{i}$ by $w_{a_{i}}$ where $\mu x_{i}=\lambda x_{i}=\theta a_{i}$. If $\mu$ and $\lambda$ are extended to $L^{\prime}$ in such a way that each $\mu w_{a_{i}}=\lambda w_{a_{i}}=\theta a_{i}$, then $\mu F=\mu D$ and $\lambda F=\lambda D$. Now, if $\mu F=1, \mu D=1$, and, since $D$ is a sentence, $D$ holds in $\theta \mathfrak{A}_{A}$, hence in $\mathfrak{U}_{A}$. Then $D$ is in $\mathfrak{A}_{A^{*}}$ and hence holds in $\mathfrak{C}^{\prime}$, whence $\lambda D=1$ and $\lambda F=1$. This establishes that $\sqrt{5}$ is an elementary extension of $\theta_{A}$.

It is now a trivial matter to construct $\mathfrak{D}$ from $\mathbb{C}$ by replacing each element $\theta a$ in $(5$ by $a$. Then $\mathfrak{D}$ is an elementary extension of $\mathfrak{A}$ itself, and the O-map of $\mathfrak{C}$ onto $\mathfrak{B}$ induces an O-map of $\mathfrak{D}$ onto $\mathfrak{B}$.

We come now to the main result concerning $Q$-maps.

Theorem 4. Let $\mathfrak{A}$ be a model of the language $L$, and $K$ an elementary class of models of $L$. Then the following are equivalent:

(1) all Q-positive sentences of $L$ that hold in $K$ also hold in $\mathfrak{A}$;

(2) some elementary extension of $\mathfrak{A}$ is a Q-image of a model in $K$.

Proof. Assume (1). Let $\Gamma=K^{*}$ and $\Delta=\mathfrak{U}_{A}^{*}$. Let $Q, Q^{\prime}$, and $I$ be as before. Let $\Delta^{\prime}$ result from $\Delta$ by replacing each relation symbol $q$ in $Q$ by the corresponding $q^{\prime}$ in $Q^{\prime}$. Suppose $\Gamma, I, \Delta^{\prime}$ inconsistent. By the Compactness Theorem, $\Gamma, I, D^{\prime} \Rightarrow 0$ where $D$ is a finite conjunction of sentences from $\Delta$, hence itself belongs to $\Delta$. Then $\Gamma, I \Rightarrow$ $\sim D^{\prime}$, and, by Proposition 2, and Compactness, there exists a $Q$-positive sentence $P$, not containing the symbols $q^{\prime}, w_{a}, e_{A}$, that is, in $L$, such that $L \Rightarrow P$ and $P \Rightarrow \sim D$. But $\Gamma \Rightarrow P$ implies that $P$ holds in $K$, and, since $P$ is a $Q$-positive sentence of $L$, that $P$ holds in $\mathfrak{A}$. Therefore $P$ holds in $\mathfrak{H}_{A}$, and $P \Rightarrow \sim D$ gives a contradiction. 
It has been shown that $\Gamma, I, \Delta^{\prime}$ is consistent, hence holds for some model $\mathbb{C}$ of the language $L^{\prime}$ obtained from $L$ by adjoining the symbols $q^{\prime}, w_{a}, e_{A}$. Let $\mathfrak{D}$ be the restriction of $\mathbb{C}$ to the language $L_{A}^{\prime}$ excluding the symbols $q$; since $\mathbb{S}$ satisfies $\Delta^{\prime}$, so does $\mathfrak{D}$. Define a model $\mathfrak{B}^{\prime}$ of $L_{A}$ to agree with $\mathfrak{D}$ except that $\mathfrak{B}^{\prime} q=\mathfrak{D} q^{\prime}$; then $\mathfrak{B}^{\prime}$ satisfies $\Delta$. By Proposition 3 , some 0 -image $\mathfrak{B}^{*}$ of the restriction $\mathfrak{B}$ of $\mathfrak{B}^{\prime}$ to $L$ is an elementary extension of $\mathfrak{A}$.

Let $\mathbb{F}$ be the restriction of $\sqrt{5}$ to $L$; since $\sqrt{ }$ satisfies $\Gamma$, so does $\mathbb{F}$, and $\mathbb{5}$ is in $K$. Now $\mathfrak{s} w=\sqrt{ } w=\mathfrak{B} w$ for all $w$ in $W$, and $\mathfrak{F} r=\sqrt{ } r r=$ $\mathfrak{B} r$ for all $r$ not in $Q$, while, for $q$ in $Q$, $\mathfrak{s} q=\mathfrak{S} q$ while $\mathfrak{B} q=\mathfrak{D} q^{\prime}=\mathfrak{S} q^{\prime}$, and, since $\mathbb{C}$ satisfies the seetences $I, \mathbb{S} q \subseteq \mathfrak{B} q$. It follows that the identity map $\theta$ on the common domain $C$ of $\mathbb{F}$ and $\mathfrak{B}$ is a $Q$-map of $\mathbb{F}$ onto $\mathfrak{B}$. It follows that the $O$-image $\mathfrak{B}^{*}$ of the $Q$-image $\mathfrak{B}$ of $\mathfrak{F}$ is a $Q$-image of $\mathfrak{F}$ : the elementary extension $\mathfrak{B}^{*}$ of $\mathfrak{A}$ is the $Q$-image of $\mathfrak{F}$ in $K$.

To show that (2) implies (1), it suffices to show that if $\mathfrak{A}$ is a $Q$ image of some $\mathfrak{B}$ in $K$, and $P$ in $\Gamma$ is $Q$-positive, then $P$ holds in $\mathfrak{A}$. Define a model $\mathbb{C}$ of the language $L^{\prime}$, obtained from $L$ by adjoining the symbols $q^{\prime}$, by taking as domain the common domain $A$ of $\mathfrak{A}$ and $\mathfrak{B}$; setting $\mathfrak{S} w$ equal to the common value $\mathfrak{A} w=\mathfrak{B} w$; for $r$ not in $Q$, setting $\mathfrak{C} r=\mathfrak{A} r=\mathfrak{B} r$; and defining $\mathfrak{b} q=\mathfrak{B} q, \mathfrak{c} q^{\prime}=\mathfrak{2} q$. Since $\mathfrak{B}$ is in $K, \mathfrak{B}$ satisfies $P$ and so does $\mathfrak{C}$. Since $\mathfrak{A}$ is a $Q$-image of $\mathfrak{B}$, each $\mathfrak{B} q \subseteq \mathfrak{A} q$, that is, each $\sqrt{ } q \subseteq \sqrt{ } q^{\prime}$, whence $\sqrt{ }$ satisfies the sentences $I$. Since $P$ is $Q$-positive, it follows by Proposition 1 that $P, I \Rightarrow P^{\prime}$, whence $P^{\prime}$ holds in $\mathfrak{C}$, and, since $\mathfrak{S} P^{\prime}=\mathfrak{U} P, P$ holds in $\mathfrak{A}$.

Corollary 4.1. An elementary class $K$ is closed under Q-maps if and only if it is the set of all models for some set of Q-positive sentences.

Proof. Assume $K$ closed under $Q$-maps. Let $K=\Gamma^{*}$, and let $I$ be the set of all $Q$-positive consequences of $\Gamma$. If $\mathfrak{A}$ is in $\Pi^{*}$, some elementary extension $\mathfrak{B}$ of $\mathfrak{A}$ is a $Q$-image of a model $\mathfrak{C}$ in $K$. But then $\mathfrak{B}$ and therefore $\mathfrak{A}$ are in $K$. Thus $\Pi^{*} \subseteq \Gamma^{*}$; since $\Pi \subseteq \Gamma$ implies $\Gamma^{*} \subseteq \Pi^{*}, K=\Gamma^{*}=I I^{*}$. The converse is immediate.

CoRollary 4.2. A set of sentences is preserved under Q-maps if and only if it is equivalent to a set of Q-positive sentences.

4. The Main Theorem. We now choose once and for all a relation symbol $e$ of rank two, and consider henceforth only languages $L$ that contain this symbol. A model $\mathfrak{A}$ of $L$ will be called a relational system provided $\mathfrak{A} e$ is the identity relation on the domain $A$ of $\mathfrak{A}$. We shall speak of the set of all relational systems in an elementary class as an elementary class of relational systems. 
The term homomorphism will be taken in the broad sense, for a map that preserves all functions and relations, that is, an $R$-map. The term projection will be used for the narrower concept of 0 -map: $\mathfrak{B}$ is (the image under) a projection of $\mathfrak{A}$ if and only if $\mathfrak{B}$ is isomorphic to a quotient system of $\mathfrak{A}$. The other component of the concept of homomorphism in contained in that of enlargement, or $R$-isomophism: $\mathfrak{B}$ is (the image under) an enlargement of $\mathfrak{A}$ if and only if $\mathfrak{B}$ is isomorphic to a system obtained from $\mathfrak{U}$ by replacing its relations by more extensive relations. It is easily seen that if $\theta$ is any homomorphism of $\mathfrak{U}$ onto $\mathfrak{B}$, then $\mathfrak{A}$ has an enlargement $\mathfrak{X}^{\prime}$, defined by taking $\mathfrak{X}^{\prime} r=\theta^{-1} \mathfrak{B} r$ for all $r$ except, $e$, such that $\theta$ induces a projection of $\mathfrak{H}^{\prime}$ outo $\mathfrak{B}$.

THEOREM 5. Let $\mathfrak{A}$ be a relational system of the language $L$, and $K$ an elementary class of systems of $L$. Then the following are equivalent:

(1) $\mathfrak{A}$ satisfies all sentences of $L$ that hold in $K$ and are

positive in all relation symbols

positive in the symbol $e$

positive in all relation symbols except $e\}$

(2) $\mathfrak{A}$ has an elementary extension that is

$\left\{\begin{array}{l}a \text { homomorphic image } \\ \text { a projection } \\ \text { an enlargement }\end{array}\right\}$ of a system in $K$.

Proof. Let $Q_{1}=R, Q_{2}=\{e\}, Q_{3}=R-\{e\}$.

If $\mathfrak{A}$ satisfies (2) it is a $Q_{i}$-image of a system in $K \cong K^{* *}$, and hence, by Theorem $4, \mathfrak{A}$ satisfies all $Q_{i}$-positive sentences in $K^{* * *}=K^{*}$.

For the converse, suppose that $\mathfrak{A}$ is a relational system that satisfies all the $Q_{i}$-positive sentences in $K^{*}$. By Theorem 4 , there exists a model (5) (not necessarily a relational system) in $K^{* *}$ and a $Q_{i}$-map $\theta$ of c onto a model $\mathfrak{B}$ that is an elementary extension of $\mathfrak{A}$. Since $K$ is a class of relational systems, $K^{*}$ contains sentences requiring that $e$ be interpreted as a congruence, whence $\mathfrak{C} e$ is a congruence on $\mathfrak{C}$. Since $\mathfrak{A}$ is a relational system, $\mathscr{A} e$ is a congruence, and, indeed, the identity on the domain $A$ of $\mathfrak{A}$. Since $\mathfrak{B}$ is an elementary extension of $\mathfrak{A}$, hence elementarily equivalent to $\mathfrak{A}, \mathfrak{B} e$ is a congruence on $\mathfrak{B}$, and its restriction to $A$ is the identity on $A$. It follows that $\mathfrak{B} / \mathfrak{B} e$ is an elementary extension of $\mathfrak{A}$.

The map $\theta$ induces a $Q_{i}$-isomorphism $\bar{\theta}$ of $\sqrt{ } / \mathbb{S} e$ onto $\mathfrak{B} / \mathbb{C} e$. Since $\mathfrak{S} e \subseteq \mathfrak{B} e$, there is a canonical projection $\kappa$ of $\mathfrak{B} / \mathfrak{C} e$ onto $\mathfrak{B} / \mathfrak{B} e$. Hence $\kappa \bar{\theta}$ is a $Q_{i}$-map of the relational system $\mathbb{E} / \mathbb{S} e$ onto the relational system $\mathfrak{B} / \mathfrak{B} e$. This completes the case of $Q_{1}=R$. For $Q_{2}=\{e\}$, $\mathbb{E}$ and $\mathfrak{B}$ differ only in their values $\mathfrak{G} e$ and $\mathfrak{B} e$, whence $\mathfrak{E} / \mathfrak{G} e=\mathfrak{B} / \mathfrak{C} e$, and $\kappa$ is a 
projection of $\mathbb{C} / \mathfrak{C} e$ onto $\mathfrak{B} / \mathfrak{B} e$. For $Q_{3}=R-\{e\}$, $\mathfrak{C} e=\mathfrak{B} e$, whence $\mathfrak{B} / \mathfrak{C} e=\mathfrak{B} / \mathfrak{B} e$ and $\theta$ is a $Q_{3}$-isomorphism, that is, an enlargement, from $\mathfrak{C} / \mathfrak{C} e$ onto $\mathfrak{B} / \mathfrak{B} e$.

It would be possible, by the same arguments, to generalize Theorem 5 to $Q$-maps, where $Q \subseteq R$ may contain $e$ or not, and indeed to maps increasing in one set $Q$ of relation symbols and decreasing in a second set $Q^{\prime}$. But, for simplicity, we shall rather restrict our attention to the entirely typical case of homomorphisms.

CoROLlaRY 5.1. Let $K$ be an elementary class of relational systems. $A$ sentence $S$ is true for all homomorphic images of systems in $K$ if and only if $S$ is a consequence of some positive sentence that holds for all systems in $K$.

Proof. If $S$ is a consequence of a positive sentence $P$ that holds for all systems in $K$, it follows by the theorem that $P$, and therefore also $S$, hold for all homomorphic images of systems in $K$. Conversely, if $S$ holds for all homomorphic images of systems in $K$, and hence for all systems having such images as elementary extension, it follows by the theorem that $S$ holds for all systems that satisfy the set $I$ of all positive sentences that hold for every system in $K$. Thus $I \Rightarrow S$, and by the Compactness Theorem $P_{1}, \cdots, P_{n} \Rightarrow S$ for some finite set of $P_{1}, \cdots, P_{n}$ in $\Pi$, whence $P \Rightarrow S$ for $P=P_{1} \wedge \cdots \wedge P_{n}$ in $\Pi$.

CoRollary 5.2. Let $K$ be an elementary class of relational systems. Every homomorphic image of a system in $K$ itself belongs to $K$ if and only if $K$ is the class of all systems satisfying a certain set of positive sentences.

Proof. Let $K=\Gamma^{*}$, and suppose that $H(K) \subseteq K$, where $H(K)$ is the class of all homomorphic images of systems in $K$. Let $I I$ be the set of all positive sentences in $\Gamma$. Since $\Pi \cong \Gamma$, it is immediate that $\Gamma^{*} \subseteq \Pi^{*}$. By Corollary 5.1, every sentence $S$ in $\Gamma$ is a consequence of some sentence $P$ in $\Pi$, whence $\Gamma^{*} \subseteq \Pi^{*}$. It follows that $\Pi^{*}=\Gamma^{*}=K$.

CoRollary 5.3. A sentence has the property that whenever it holds for a system $\mathfrak{A}$ it holds for every homomorphic image of $\mathfrak{A}$ if and only if it is equivalent to a positive sentence.

Proof. In Corollary 5.2, take $K$ to be the class characterized by a single sentence.

If a relational system $\mathfrak{A}$ satisfies the set of all positive sentences true for a system $\mathfrak{B}$, it follows from the theorem, with $K=\mathfrak{B}^{* *}$, that some elementary extension $\mathfrak{A}^{\prime}$ of $\mathfrak{A}$ is a homomorphic image of a system 
$\mathfrak{B}^{\prime}$ that is elementarily equivalent to $\mathfrak{B}$. In fact, by passing from the originally given language $L$ to the language $L_{B}$, there is no difficulty in establishing a stronger proposition, as follows: $\mathfrak{A}$ satisfies all positive sentences true for $\mathfrak{B}$ if and only if $\mathfrak{A}$ has an elementary extension $\mathfrak{Y}{ }^{\prime}$ and $\mathfrak{B}$ an elementary extension $\mathfrak{B}^{\prime}$ such that $\mathfrak{Y}^{\prime}$ is a homomorphic image of $\mathfrak{B}^{\prime}$.

Let $H$ an elementary class of relational systems, and call a system in $H$ an $H$-system. The following relativized version of Theorem 5 is contained directly in that theorem.

Theorem 5'. If $\mathfrak{2}$ is an $H$-system and $K$ an elementary class of $H$-systems, then the following are equivalent:

(1) $\mathfrak{A}$ satisfies all positive sentences that hold in $K$;

(2) $\mathfrak{A}$ has an elementary extension that is a homomorphic image of a system in $K$.

The relativized forms of the corollaries now follow as before, provided the relation $P \Rightarrow T$ is replaced by that of $H$-implication: $H, P \Rightarrow T$, and equivalence by H-equivalence. As an example, the relativized version of Corollary 5.3 asserts the equivalence of the following properties of a first order sentence $S$ of group theory:

(1) if $\mathfrak{A}$ and $\mathfrak{B}$ are torsionfree groups, if $\mathfrak{B}$ is a homomorphic image of $\mathfrak{A}$, and $S$ holds for $\mathfrak{A}$, then $S$ holds for $\mathfrak{B}$;

(2) there exists a positive sentence $P$ such that, for each torsionfree group $\mathfrak{A}, S$ holds if and only if $P$ holds;

and hence, further,

(2) there exists a positive sentence $P$ such that the equivalence of $S$ and $P$ follows from the axioms for torsionfree groups.

5. Complementary examples. We first note that the conclusion of Theorem 5 does not follow without the requirement that the class $K$ be elementary. For this, let $L$ be the language of elementary identity theory, without operation symbols and without relation symbols other than $e$. The relational systems for this language are simply sets. Let $K$ be the class of all finite systems; clearly $H(K) \subseteq K$. It is well known and easily seen that $K^{*}$ consists only of those sentences that are true in all models. A fortiori, every system, infinite or finite, satisfies all positive sentences that hold for $K$. But an elementary extension of an infinite system is itself infinite, hence cannot belong to $H(K)$.

Next we show that, even if $K$ is elementary, the class $H(K)$ of all homomorphic images of systems in $K$ need not be elementary; hence the reference to elementary extensions in Theorem 5 can not be deleted. For this, let $L$ contain no operation symbols, and only a single binary relation symbol $r$ in addition to $e$. Let $S$ be the following sentence: 


$$
S=\exists x \exists y \forall z \exists t: r(x, y) \wedge \cdot r(x, z) \supset r(x, t) \wedge r(z, t) .
$$

For $n=1,2, \cdots$, let $S_{n}$ be the following sentence:

$$
\begin{gathered}
S_{n}=\exists x_{0} x_{1} \cdots x_{n} \cdot r\left(x_{0}, x_{1}\right) \wedge r\left(x_{0}, x_{2}\right) \wedge \cdots \wedge r\left(x_{0}, x_{n}\right) \\
\wedge r\left(x_{1}, x_{2}\right) \wedge r\left(x_{2}, x_{3}\right) \wedge \cdots \wedge r\left(x_{n-1}, x_{n}\right) .
\end{gathered}
$$

We shall establish the following:

If $S \Rightarrow P$, and $P$ is positive, then $S_{n} \Rightarrow P$ for some $n=1,2, \cdots$; hence, defining $K=S^{*}, H(K)^{*}=\left\{S_{1}, S_{2}, \cdots\right\}^{* *}$.

We use the Main Theorem of [7], with $S^{1}=S$ and $S^{2}=\sim P$. The Skolem matrix $M^{1}$ of $S$ has the form

$$
r\left(s_{0}, s_{1}\right) \wedge \cdot r\left(s_{0}, z\right) \supset r\left(s_{0}, s(z)\right) \wedge r(z, s(z)),
$$

where $s_{0}, s_{1}$ are Skolem functions of rank 0 , and $s$ of rank 1, in an extension $L^{\prime}$ of $L$. The Skolem matrix $M^{2}$ of $\sim P$ is negative. If $N^{1}, N^{2}$ are the Skolem matrices of $U^{1}, U^{2}$, as in the Main Theorem of [7], evidently $N^{2}$ is negative, whence $N^{1}$ and hence $U^{1}$ are positive. We have that $U^{1} \Rightarrow P$, and, from the relation of $U^{1}$ to $S^{1}$, that $U^{1}$ follows from a universal sentence with positive matrix $M$, where $M$ follows by propositional calculus alone from a set $\Sigma$ of instances of $M^{1}$. Define a sequence of terms $t_{0}, t_{1} \ldots$ in $L^{\prime}$ by setting $t_{0}=s_{0}, t_{1}=s_{1}$, and, inductively, $t_{n+1}=s\left(t_{n}\right)$ for all $n \geqq 1$. Define a substitution $\chi$ on the atomic formulas $F$ of $L^{\prime}$ by setting $\chi F=F$ if $F$ is $r\left(t_{n}, t_{n+1}\right)$ or $r\left(t_{0}, t_{n+1}\right)$, for some $n=0,1,2, \cdots$, and setting $\chi F=0$ otherwise. Since $M$ is positive and each $\chi F \Rightarrow F, \chi M \Rightarrow M$. Since $\Sigma \Rightarrow M$ by propositional calculus, $\chi \Sigma \Rightarrow \chi M$. Thus $\chi \Sigma \Rightarrow M$. Now $\chi \Sigma$ is evidently equivalent to the set of all formulas $r\left(t_{0}, t_{n+1}\right)$ and $r\left(t_{n}, t_{n+1}\right)$, whence, by the Compactness Theorem, $M$ is a consequence of a finite set of them, and hence, for some $n$, of

$$
r\left(t_{0}, t_{1}\right) \wedge \cdots \wedge r\left(t_{0}, t_{n}\right) \wedge r\left(t_{1}, t_{2}\right) \wedge \cdots \wedge r\left(t_{n-1}, t_{n}\right) .
$$

But now $U^{1}$, which follows from $M$, follows equally from the Skolem matrix of $S^{n}$, hence from $S^{n}$ itself, and $S^{n} \Rightarrow P$.

Let $\mathfrak{A}$ be a relational system for $L$. The sentence $S$ evidently requires that the domain $A$ of $\mathfrak{A}$ contain an infinite chain of elements, not necessarily distinct, $a_{0}, a_{1}, \cdots$, such that $\mathfrak{A} r\left(a_{0}, a_{n}\right)$ and $\mathfrak{A} r\left(a_{n-1}, a_{n}\right)$ for all $n \geqq 1$. Since the image of such a chain in any homomorphic image of $\mathfrak{A}$ is again such a chain, every $\mathfrak{A}$ in $H(K)$ contains such a chain. On the other hand, each condition $S_{n}$ requires of a system $\mathfrak{A}$ that it contain a finite chain $a_{0}, a_{1} \cdots, a_{n}$ related in this fashion, whence a system $\mathfrak{A}$ is in $H(K)^{*}$ if it contains such chains of unbounded lengths. If $\mathfrak{A}$ is a system with domain $A=\left\{a_{0}, a_{1}, \cdots\right\}$ and $\mathfrak{U} r\left(a_{i}, a_{j}\right)$ is true if and only if $j<i$, evidently $\mathfrak{A}$ contains chains $a_{n}, a_{n-1}, \cdots, a_{0}$ for all $n$, 
but no infinite chain of the kind required by $S$, whence $\mathfrak{A}$ is in $H(K)^{*}$ but not in $H(K)$.

We conclude by showing that it is not in general decidable whether a sentence of a first order language is equivalent to some positive sentence. ${ }^{5}$ A first order theory $T$ may be taken as consisting of a language $L$ together with a consistent logically closed set $\Gamma=\Gamma^{* *}$ of sentences of $L$, the theorems of $T$. The theory $T$ is undecidable if there is no effective method of deciding, for all sentences $S$ of $L$, whether $S$ is a theorem of $T$, that is, if the set $\Gamma$ is not recursive; this concept is of interest primarily in the case that there exists a finite, or at least recursive, set $\Gamma_{0}$ of axioms, such that $\Gamma_{0}{ }^{* *}=\Gamma$. We shall confine our attention to finitely axiomatizable undecidable theories that have the following additional property:

$\left(^{*}\right)$ every model in which $\Gamma$ holds has as homomorphic image some one-element model in which $\Gamma$ holds.

Two important examples of such theories are the following:

(1) $L$ contains at least one relation symbol (other than the identity symbol) of rank greater than one, $\Gamma$ empty; ${ }^{6}$

(2) $L$ contains the identity symbol and an operation symbol $w$ of rank 2 , and $\Gamma$ is a set of axioms for group theory with $e$ interpreted as equality and $w$ as the group composition. ${ }^{7}$

Let $\Sigma$ be the set of all sentences $S$ of $L$ such that $S$ holds in every one-element model in which $\Gamma$ holds. Clearly $\Gamma \cong \Sigma$. Moreover, it is easily decidable, for $C$ the conjunction of all axioms in $\Gamma_{0}$, whether $C \supset S$ holds in all one-element models, and hence whether $S$ is in $\Sigma$. Consequently, it is not decidable whether a sentence in $\Sigma$ is a theorem.

Let $S$ be in $\Sigma$. Suppose first that $S$ is a theorem. Then $\Gamma \Rightarrow S$, whence $\sim S$ is $T$-equivalent to the false sentence 0 , which is positive; that is, $\Gamma \Rightarrow \sim S \supset 0 \cdot \wedge \cdot 0 \supset \sim S$. Suppose now that $S$ is not a theorem. Then there exists a model $\mathfrak{A}$ in which $\Gamma$ holds while $S$ fails, and hence $\sim S$ holds. In view of the assumption $\left(^{*}\right) \mathfrak{A}$ has as homomorphic image some one-element system $\mathfrak{B}$ in which $\Gamma$ holds. Since $S$ is in $\Sigma, S$ holds in $\mathfrak{B}$, that is, $\sim S$ fails in $\mathfrak{B}$. Since $\Gamma$ and $\sim S$ both hold in $\mathfrak{A}$, while $\Gamma$ holds and $\sim S$ fails in the homomorphic image $\mathfrak{B}$ of $\mathfrak{A}$, it follows from Theorem $5^{\prime}$ that $\sim S$ is not $T$-equivalent to any positive sentence. We have shown that, for $S$ in $\Sigma, \sim S$ is equivalent to a positive sentence (and, indeed, to the positive sentence 0 ) if and only if $S$ is a theorem. It follow that there exists no effective method of deciding, for sentences $S$ such that $\sim S$ is in $\Sigma$, nor, therefore, for all sentences of $L$, whether $S$ is $T$-equivalent to a positive sentence.

5 For the main concepts of this paragraph, see [14].

${ }^{6}$ See Church [1].

7 For the undecidability of the elementary theory of groups, see [14, p. 84] and the reference to Tarski given there; see also [10]. 


\section{REFERENCES}

1. A. Church, A note on the Entscheidungsproblem, J. Symbolic Logic 1 (1936), 40, 101.

2. L. Henkin, The completeness of the first order functional calculus, J. Symbolic Logic 14 (1949), 159.

3. A. Horn, On sentences which are true of direct unions of algebras, J. Symbolic Logic 16 (1951), 14.

4. H. J. Keisler, J. Symbolic logic, to appear.

5. J. Łoś, Sur les classes définissables, p. 107, in Mathematical Interpretation of Formal Systems, North Holland, Amsterdam, 1955.

6. R. C. Lyndon, review of [3], J. Symbolic Logic 16 (1951), 216.

7. R. C. Lyndon, An interpolation theorem in the predicate calculus, Pacific J. Math., $\mathbf{9}$ (1959),

8. A. I. Malcev, Subdirect products of models, Dokl. Akad. Nauk SSSR (N.S.) 109 (1956), 264.

9. E. Marczewski, Sur les congruences et les proprietés positives d'algèbres abstraites, Colloq. Math. 2 (1951), 220.

10. P. S. Novikov, On the algorithmic unsolvability of the word problem in group theory, Trudy Math. Inst. Steklov, No. 44, Moscow, 1955.

11. A. Robinson, On the Metamathematics of Algebra, North Holland, Amsterdam, 1951.

12. A. Tarski, Some notions and methods on the borderline of algebra and metamathematics, Proc. International Congress of Math. 1, Cambridge, 1950, 705.

13. A. Tarski, Contributions to the theory of models, I, II, III, Indag. Math. 16 (1954), 572, 582; 17 (1955), 55.

14. A. Tarski, with A. Mostowski and R. M. Robinson, Undecidable Theories, North Holland, Amsterdam, 1953.

15. A. Tarski and R. L. Vaught, Arithmetical extensions of relational systems, Comp. Math. 13 (1957), 81.

UNIVERSITY OF MICHIGAN 


\section{PACIFIC JOURNAL OF MATHEMATICS}

\section{EDITORS}

\section{David Gilbarg}

Stanford University

Stanford, California

R. A. Beaumont

University of Washington

Seattle 5 , Washington
A. L. Whiteman

University of Southern California

Los Angeles 7, California

L. J. PAIGE

University of California

Los Angeles 24, California

\section{ASSOCIATE EDITORS}
E. F. BECKENBACH
C. E. BURGESS
E. HEWITT
A. HORN

\author{
V. GANAPATHY IYER \\ R. D. JAMES \\ M. S. KNEBELMAN \\ L. NACHBIN
}
I. NIVEN
E. G. STRAUS
T. G. OSTROM
H. L. ROYDEN
G. SZEKERES
M. M. SCHIFFER
F. WOLF
K. YOSIDA

\section{SUPPORTING INSTITUTIONS}

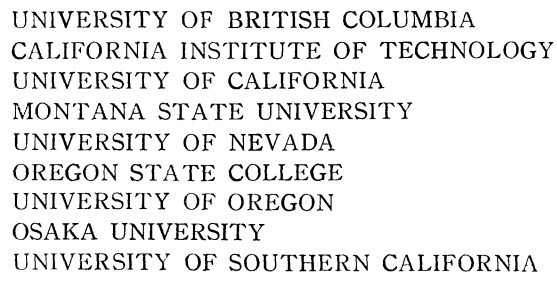

UNIVERSITY OF BRITISH COLUMBIA CALIFORNIA INSTITUTE OF TECHNOLOGY UNIVERSITY OF CALIFORNIA MONTANA STATE UNIVERSITY UNIVERSITY OF NEVADA OREGON STATE COLLEGE UNIVERSITY OF OREGON OSAKA UNIVERSITY UNIVERSITY OF SOUTHERN CALIFORNIA

STANFORD UNIVERSITY

UNIVERSITY OF TOKYO

UNIVERSITY OF UTAH

WASHINGTON STATE COLLEGE

UNIVERSITY OF WASHINGTON

AMERICAN MATHEMATICAL SOCIETY CALIFORNIA RESEARCH CORPORATION HUGHES AIRCRAFT COMPANY SPACE TECHNOLOGY LABORATORIES

Printed in Japan by Kokusai Bunken Insatsusha (International Academic Printing Co., Ltd.), Tokyo, Japan 


\section{Pacific Journal of Mathematics}

\section{Vol. 9, No. 1 \\ May, 1959}

Julius Rubin Blum and Murray Rosenblatt, On the structure of infinitely

divisible distributions . ............................. 1

Robert Geroge Buschman, Asymptotic expressions for

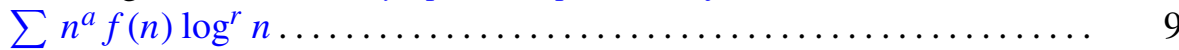

Eckford Cohen, A class of residue systems $(\bmod r)$ and related arithmetical

functions. I. A generalization of Möbius inversion .............. 13

Paul F. Conrad, Non-abelian ordered groups ................... 25

Richard Henry Crowell, On the van Kampen theorem............... 43

Irving Leonard Glicksberg, Convolution semigroups of measures ........ 51

Seymour Goldberg, Linear operators and their conjugates ............ 69

Olof Hanner, Mean play of sums of positional games .............. 81

Erhard Heinz, On one-to-one harmonic mappings ................ 101

John Rolfe Isbell, On finite-dimensional uniform spaces . . ........... 107

Erwin Kreyszig and John Todd, On the radius of univalence of the function

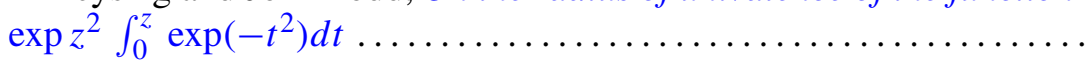

Roger Conant Lyndon, An interpolation theorem in the predicate

calculus......................................... 129

Roger Conant Lyndon, Properties preserved under homomorphism ........ 143

Roger Conant Lyndon, Properties preserved in subdirect products ....... 155

Robert Osserman, A lemma on analytic curves ................ 165

R. S. Phillips, On a theorem due to Sz.-Nagy..................... 169

Richard Scott Pierce, A generalization of atomic Boolean algebras ....... 175

J. B. Roberts, Analytic continuation of meromorphic functions in valued fields................................. 183

Walter Rudin, Idempotent measures on Abelian groups ................ 195

M. Schiffer, Fredholm eigen values of multiply-connected domains ........ 211

V. N. Singh, A note on the computation of Alder's polynomials ......... 271

Maurice Sion, On integration of 1-forms ...................... 277

Elbert A. Walker, Subdirect sums and infinite Abelian groups........... 287

John W. Woll, Homogeneous stochastic processes . .................. 293 